\title{
Contact area predicts surgical outcome
}

A new method that uses CT imaging to calculate the contact surface area (CSA) between a kidney tumour and the adjacent renal parenchyma enables prediction of the outcomes and complications of partial nephrectomy.

The anatomical location, size and complexity of renal masses can all affect surgical outcomes, and various systems are currently in use to objectively quantify these variables, to determine whether nephron-sparing surgery is appropriate. CSA summarizes tumour operative complexity in a single measure that combines the size of the renal mass with the degree of intraparenchymal extension.

CSA has been validated using preoperative CT images for 162 consecutive male and female patients undergoing minimally invasive partial nephrectomy. 3D image-rendering software enabled estimations of tumour size (mean $3.1 \mathrm{~cm}$ ) and calculation of CSA (mean $18.3 \mathrm{~cm}^{2}$ ). Baseline patient demographics were similar for low CSA $\left(<20 \mathrm{~cm}^{2}, n=104\right)$ and high CSA $\left(>20 \mathrm{~cm}^{2}, n=58\right)$. On multivariable logistic regression analysis, high CSA was significantly associated with operative time $\geq 4$ hours (OR 4.67), complications (OR 4.08), length of stay $\geq 4$ days (OR $3.71)$ and $\geq 10 \%$ decrease in estimated glomerular filtration rate 1 month after surgery (OR 3.51). By contrast, a currently used nephrometric measure (PADUA score $\geq 10$ ) was not significantly associated with any of these parameters.

The strength of the CSA measure could be that it is derived from just the two most relevant tumour features. It is easy to calculate, if appropriate imaging software is available, and it seems to also correlate with pathological tumour features. With further validation, this measure could be added to the nephrometry scoring systems used to assess patients prior to nephron-sparing surgery for kidney cancer.

\section{Robert Phillips}

Original article Leslie, S. et al. Renal tumor contact surface area: a novel parameter for predicting complexity and outcomes of partial nephrectomy. Eur. Urol. doi:10.1016/ j.eururo.2014.03.010 